Rev. Elev. Méd vét. Pays trop, 1965, 18, 2 (175-181)

\title{
Intoxication accidentelle de bovins par douchage avec un insecticide organo-phosphoré, le carbophenothion
}

\author{
par G. UILENBERG et R. GAULIER
}

\begin{abstract}
RÉSUMÉ
Par sulte d'une erreur, 21 bovins âgés de 6 mors à 2 ans ont été douchés 2 fois par semaine, pendant presqu'un mois, au carbophénothion à une concentration de 0,1 p. 100.18 bovins sont devenus malades, après 4 à $B$ douchages, 9 d'entre eux sont morts.

Les symptômes les plus frappants de cette intoxication à évolution lente consistaient en une diarrhée profuse, une salıvation excessive et une démarche raide. La seule lésion conslante à l'autopsie élait un œedème des poumons. L'âge en soi semble moins important pour la sensibilité individuelie que la taılle et la condition physique. Un chaton est mort le lendemain d'un saupoudrage avec du carbophénothion à 1 p. 100 dans du talc. Les résultats des détermınations de la cholinestérase sanguıne sont rapportés, aınsı que quelques remarques sur le traitement. II n'y avait pas de corrélation netle entre l'impartance des symptômes et celle de la baisse de l'activité de la cholinestérase. Les auteurs mettent en garde contre l'emploi sur les animaux d'insecticides dont on connaît insuffisamment la toxıcité à long terms
\end{abstract}

Le Laboratoire Central de l'Elevage à Tananarive possède une étable spécıalement aménagée pour maintenir des bovins d'expérience à l'abri des tiques. Pour renforcer la protection, les animaux sont douchés deux fois par semaine au carbaryl (1-naphthy|-N-méthyl carbamate $=$ Sevin R), à une concentration de 0,425 p. 100. Plusieurs dizaines de bovins ont aıns। été traıtés pendant des mois, quelques-uns pendant deuxans, sans que jamals des symptômes d'intoxication ne soient apparus, même lorsqu'll s'agissait de veaux douchés dès la naissance. Ceci confirme les données bibliographiques (qui ne seront pas énumérées $(\mathrm{ci})$, ainsi que les résultats de nos quelques expériences sur la toxicıté du carbaryl (Ropport Annuel, 1962), qui indiquent sa très faible toxicıté pour les anımaux à sang chaud.

Le laboratoire ayant épuisé son stock de carbaryl en novembre 1964, un nouveau sac de ce produit, d'une marque employée souvent auparavant, est commandé.

Quelques semaines plus tard, la plupart des animaux de l'étable anti-tiques sont en mauvaise condition physıque; quelques-uns ontune diarrhée profuse, les yeux enfoncés dans les orbites, une salivation excessive et une démarche raide. L'état de ces animaux s'aggrave pendant les jours qui suivent, les mềmes symptômes se déclenchent chez d'autres, et deux veaux sont sacrifiés in extremis, tandis que d'autres succombent, malgré divers traitements (oxytétracycline, sulfaguanidine, salicylate basique d'alumıne, ration alimentaıre plus élevée), et sans que la cause de la maladie puisse être déterminée. Pendant cette période, les animaux sont toujours douchés deux fols par semane, jusqu'au jour où notre attention est attırée sur le fait que la Masson qui avait livré I'ınsecticide, a fait une 
erreur : le dernier sac acheté ne contient pas une poudre movillable de carbaryl à 85 p. 100 , sans autre insecticide, mais une poudre mouillable contenant un mélange de 40 p. 100 de carbaryl et de 20 p. 100 de carbophénothion (=0,0-diéthyl $\quad S$-( $p$-chlorophénylthio)-méthyl phosphorodithioate $=$ Trithion $R$ ), insecticide organophosphoré de toxicité importante. Le nom commercial de ce mélange est le même que celui du carbaryl seul; la composition réelle n'est donnée qu'en très petites lettres sous ce nom.

La cause de la maladie est trouvée.

Quelques données sur la toxictté du carbophénothion pour le rat, exprimée en DL 50 $(\mathrm{mg} / \mathrm{kg})$ :

BRADY e.a. (1960) : 18 (voie orale), GAINES (1960) : 30 (vole orale, rał mâle), 10 (vore orde, femelle), 54 (voie cutanée, rał mâle), 27 (voie cutanée, femelle),

WYNIGER (1962) : 28 (voie orale), BRODEUR et

DUBOIS (1963) : 11 (voie intrapéritonéale, jeunes rats de 3 semaines, 40 (vole intrapéritonéale, rat mâle).

Ces résultats placent donc le carbophénothion parmi les insecticides de très grande toxicité. (Notons que, d'après TILEMANS (1957), il n'est autorisé aux Etats-Unis d'Amérique que pour les plantes non comestibles, vu sa toxicıté.)

Plus intéressantes pour notre cas sont les expériences de YOUNGER e.a. (1963), qui ont étudié la toxicité du carbophénothion pour les bovins, appliqué en douchage, avec une suspension préparée à partir d'une poudre mouillable, comme ce fut notre cas. Un douchage à des concentrations de $0,05,0,1$ et 0,5 p. 100 était toxique pour des vequx âgés de 1 à 2 semaines; l'intoxication a été mortelle pour un des quatre vecux douchés à 0,05 p. 100 et pour un des trois veaux douchés à 0,1 p. 100 ; l'activité de la cholinestérase sanguine était abolssée sur tous ces animaux. Des bovıns âgés d'un à deux ans n'étalent pas intoxiqués par un seul douchage à 0,05 p. 100 et à 0,1 p. 100 , mais l'activité de la cholınestérase était abaissée sur un des deux animaux douchés à 0,1 p. 100 ; un animal douché à 1 p. 100 montraił des symptômes d'intoxication. Les symptômes observés par ces auteurs étaient: salivation excessive, diarrhée, démarche raide.

Nous donnons ci-dessous quelques détails sur les circonstances de l'intoxication à notre étable, sur les symptômes et lésions observés, sur le traitement, et sur les résultats de la détermınation de l'activité de la cholinestérase sanguine.

Le douchage au mélange de carbaryl et de carbophénothion a commencé dans la première semaine de novembre 1964. La concentration était de $5 \mathrm{~g}$ de produit par l, correspondant à une teneur en carbophénothion de 0,1 p. 100 (la poudre mouillable en contenant 20 p. 100). Cetfe suspension a été appliquée à raison d'environ 4 I par anımal, deux fols par semaine, dans la période allant de début novembre au 1 er décembre, soit un total de 8 ou 9 traitements.

Le 3 décembre, jour auquel l'erreur a été découverte, tous les animaux ont été lavés avec un détergent (Teepol (R) à 1 p. 1000), et les bovins les plus atteints ont été traités à l'atropine seule, ou combinée au Contrathion ( $R$ ) et au Diparcol $(R)$. (Ces deux derniers produits n'étaient disponibles qu'en petite quantité, et les doses employées étaıent faibles).

Il y avait, au début de novembre, 21 bovins d̀ l'étable anti-tiques.

Its peuvent être divisés en 3 groupes:

I. Bovins chez lesquels aucun symptôme caractérıstique de l'intoxication (diarrhée, salivation excessive, démarche raide), n'a été abservé.

II. Bovins ayant montré des symptômes caractérıstıques, mais ayant guéri, avec ou sans traitement.

III. Bovins chez lesquels l'intoxication a été mortelle.

\section{Groupe I.}

3 animaux, âgés au débuł de novembre de 2 ans, de 19 mols et de 10 mols, pesant respectivement environ 300,250 et $200 \mathrm{~kg}$. Tous les trois étaient alors en bonne condition physique. Ils n'ont montré aucun symptôme clinique, sauf un léger amaigrissement.

\section{Groupe II.}

9 anımaux, âgés de 6 à 14 mois, et ne pesant que de 80 à $120 \mathrm{~kg}$ environ; ils étalent pour la plupart en assez mauvaise condition physique, à la suite de splénectomies récentes ou d'inocu- 
lations d'hématozoaires pathogènes. Les premıers symptômes caractéristiques d'intoxication sont apparus sur les anımaux de ce groupe après 7 ou 8 douchages.

3 animaux, peu atteints, ont guéri sans traitement.

Les 6 autres, plus sévèrement intoxiqués, ont été traités (voir plus loin).

a) 4 d'entre eux étaient atteints au point de rester couchés, épuisés, 3 à 6 jours après les premiers symptômes ; 2 ont pu tenir debout le lendemain du début du traitement, les 2 autres seulement 4 et 6 jours plus tard.

b) 2 veaux montraient des symptômes typıques (diarrhée, hypersalivation, démarche raıde), mais n'étaient pas encore prostrés.

\section{Groupe III.}

9 animaux, âgés de 12 d̀ 14 mois, pesant environ 75 à $130 \mathrm{~kg}$ et étant pour la plupart en assez mauvaise condition physique (splénectomies récentes, inoculations d'hématozoaires pathogènes). Les premiers symptômes caractéristiques sont apparus après 4 à 7 douchages.

a) 5 des veaux sont morts avant que la cause de la maladie $n$ 'ait été découverte, dont 2 sacrifiés in extremis 4 et 8 jours après l'apparition des symptômes ; les 3 autres ont succombé, dans chaque cas 7 jours après que les premiers symptômes ont été observés.

b) 4 veaux étaient prostrés depuis 1 à 7 jours au début du traitement; 3 d'entre eux sont morts, maigré le traitement, 10 à 13 jours après les premiers symptômes, 1 à 7 jours après le début du traıtement. La mort du 4e, 4 jours après les premiers symptômes, un jour après le traitement, était complıquée par une babésiellose (Babesia argentino), suite d'une inoculation effectuée 2 semaines auparavant, à laquelle il n'avalt pas réagi par une hyperthermie; le cerveau et d'autres organes internes renfermaient de nombreuses Babesiae.

Outre les animaux dont nous venons de parler, d'autres bovins du laboratorre ont été douchés au mélange de carbaryl et de carbophénothion :

Une vache et son veau né ie 24 novembre 1964, qui devalent être protégés contre les infections transmises par les tiques en attendant d'avoir de la place à l'étable spécıale, ont été douchés 3 fois (le $25 / 11$, le $28 / 11$ et $1 / 12$ ) avec la même suspension contenant 0,1 p. 100 de carbophénothion. Une démarche raide a été observée chez le veau dès le premier douchage, mais sans autre symptôme; il a reçu par mesure de précaution, le $3 / 12,10 \mathrm{mg}$ d'atropine. La mère présentait une anorexie le $2 / 12$, maıs s'est remise sans traltement.

4 autres bovins du Laboratoire, dont un âgé de deux mois, les autres adultes, ont été douchés une fois par semaine entre le début de novembre et le 3 décembre ( 4 fois en tout), avec une concentratıon du mélange correspondant à 0,05 p. 100 de carbophénothion. Ils n'ont pas montré de symptôme d'intoxication.

Un chaton, âgé d'environ un mois, a été traité contre les puces le 2/12, avec une poudre contenant 5 p. 100 du mélange carbaryl-carbophénothion dans du talc (donc 1 p. 100 de carbophénothion), avant que l'erreur de livraison n'alt été découverte. L'animal, en bonne santé le jour du traitement, mourut le lendemain dans l'aprèsmidi. (Des chatons avaient souvent été traités avec du carbaryl à 5 p. 100 dans du talc, sans accidents.)

Un chien adulte, berger allemand, a été saupoudré avec la même poudre à 3 reprises, sans être intoxiqué.

\section{Symptômes et lésions observés :}

Les symptômes les plus frappants consistaient en une diarrhée profuse, une salivation excessive et une démarche raide, ainsi que l'ont du reste décrit YOUNGER e.a. (1963). En même temps les bovins intoxiqués maigrissaient rapidement, fait causé, au moins en partie, par l'anorexie totale et par la diarrhée. Venaient par la sulte une grande faıblesse musculare et prostration. Sur plusieurs veoux, un larmoiement a été observé, qui devenalt quelquefors purulent, peu avant la mort; quelques cas d'opacification de la cornée on été constatés. Les veaux prostrés présentaient souvent des tremblements musculaires, les mouvements du cou devenaient incoordonnés et, vers la fin, celui-cl étalt contracturé latéralement.

La température restait normale pendant l'évolution de la maladie, pour s'abaıser progressivement dans les derniers jours avant la mort, afteignant parfois jusqu'à 34 à $35^{\circ}$. Le pouls étalt souvent légèrement accéléré. 
A l'autopsie, un œè̀me important des poumons était toujours présent (seule lésıon consłante), quelquefois une légère entérite, rarement des pétéchies sur le cœur; une congestion du foie et une augmentation du volume des reins, comme le signalent YOUNGER e.a., n'ont pas été remarquées. Le cadavre était toujours étique.

\section{Traitement.}

A part le lavage de la peau au Teepol, les veaux les plus malades ont été traités avec de l'atropine, pour combattre les symptômes du système parasympathique (environ 25 à $30 \mathrm{mg}$ par $100 \mathrm{~kg}$ de poids), appliquée sur la plupart par voie sous-cutanée (puisqu'il s'agissait d'une intoxication à évolution lente), sur 3 anımaux par injection intraveıneuse. L'effet était rapide ; dans chaque cas la diarrhée ef la salivation avaient disparu le lendemain.

Les bovins les plus atteints ont, de plus, reçu de faibles doses de Contrathion (méthylfsulfate de méthyl-1 N-hydroxyımino-méthyl-2 pyridinium, réactivateur de la cholinestérase) et de Diparcol (Chlorhydrate de diéthazine qui agirait sur les effets nicotiniques des organo-phosphorés), respectivement $200 \mathrm{mg}$ et $250 \mathrm{mg}$ par animal de $100 \mathrm{~kg}$, en injection intramusculaıre.

Les traitements à l'atropine, au Diparcol et au Contrathıon ont été répétés sur certaıns animaux le lendemain ; notre stock de Contrathıon étant alors épuisé, quelques veaux ont encore reçu, le jour suivant, l'atropine seule ou en combinaison avec le Diparcol.

Les doses de Contrathion et de Diparcol étalent faibles; aussi, il n'est pas possible de juger de leur effet sur les symptômes cliniques et sur la réactivation de la cholinestérase sanguine. L'atropine a certainement sauvé la vie à quelques animaux ef l'a tout au moins prolongée pour d'autres, mais elle intervenait trop tard pour 4 veaux. La voie sous-cutanée ne semblait pas donner de résultats différents de ceux obtenus par la voie intraveineuse, sans doute parce qu'il s'agissalt d'une intoxication à évolution lente.

\section{Sensıbilité indıviduelle à l'ıntoxicatıon.}

Les 3 anımaux les plus grands (groupe I) se sont montrés les plus résistants, bien que l'un d'entre eux fôt plus jeune (10 mois), que d'autres ayant subi une intoxication grave, même mortelle, mais pesant beaucoup moins, et n'étant pas en bonne condition. Il nous a semblé que la taille et la condition physique ont jové un rôle plus important pour la sensibilité individuelle que l'âge en soi. Un veau de 6 mois, bien développé, n'a subi qu'une légère intoxication, guérie sans traitement (bien que le taux de la cholinestérase sanguıne fût extrêmement bas); d'autres de 14 mois en mauvais état physique, sont morts. Le petit veau, douché 3 fois pendant la première semaine de sa vie, n'a subi qu'une légère intoxication (démarche raide, sans diarrhée, sans salivation excessive), et guérit après une seule injection de $10 \mathrm{mg}$ d'atropine; il était bien développé à la naissance (le taux de cholinestérase était également très bas dans ce cas).

La splénectomie ne semble pas avor eu d'influence appréciable : tous les bovins à l'étable anti-tıques étaient splénectomısés, sauf un de groupe I, un de groupe II, et deux de groupe III.

\section{Activité cholinestérasique sanguine.}

Les intoxications par les insecticides organophosphorés provoquant des baisses importantes de l'actıvité cholinestérasique des tissus, celle-cl a été déterminée d'une façon systématique sur le sang des animaux intoxiqués survivants, et son évolution a été suivie pendant une pérıode de plusieurs semaines.

La technique utilisée est celle que CHARY e.a. (1961) ont adaptée au titrage des cholinestérases sanguines des bovins (évaluation du pouvoir hydrolysant des hématies vis-à-vis de l'acétylchaline, d'après la quantité d'acide acétıque mise en liberté). Pour effectuer cette mesure, différents procédés peuvent être utilıses. La méthode déterminant l'abalssement $\mathrm{du} \mathrm{pH} d u$ milieu (avec utılisation d'un $\mathrm{pH}$-mètre) a été adoptée.

Le titrage a été effectué sur un hémolysat au $1 / 100$, en présence de tampon barbital sodique et chlorure de potassium, la solution de substrat étant constituée par une solution de chlorure d'acétylcholıne à 1 p. 100, et en opérant à la température de 370 .

Un sang donnant une baisse de $\mathrm{pH}$ de 1, 2 unités en $30 \mathrm{mn}$, après correction de la baisse de $\mathrm{pH}$ imputable à l'hydrolyse spontanée de l'acétylcholine dans les mêmes conditions, a été consldéré comme normal (activité de 100 p. 100). 
Le tableau ci-après indıque les résultats des analyses.

Ces résultats semblent bien confirmer le fait (déjà sıgnalé par d'autres auteurs), qu'il n'y a pas une corrélation absolue entre la gravité des symptômes abservés et la diminution de l'activité de la cholınestérase sanguine.

Remarque : L'insecticide a temporairement été remplacé par l'H. C. H. à 0,02 p. 100 d'isomère gamma, appliqué deux fois par semaine. Le douchage au carbaryl à 0,425 p. 100 a été repris dans la quatrième semaine après le dernier traltement au carbophénothion. Le carbaryl ne semble pas avoir eu d'influence appréciable sur la cholinestérase sanguine.

\section{Discussion.}

Il existe actuellement un nombre considérable d'insecticides dont la toxicité pour les animaux à sang chaud est plus ou moins importante ; certains conviennent pour le traitement du bétall, d'autres sont trop toxiques. II nous semble que les indications portées sur les emballages ne font pas toujours ressortır suffisamment le danger que présentent certains prodults; de plus, deux formules très différentes ne devraient jamais porter le même nom commercial, comme dans le cas présent.

En ce qui concerne les insecticides utilisés sur le bétail, il convient d'être extrêmement prudent,

\begin{tabular}{|c|c|c|c|c|c|c|c|c|c|c|}
\hline \multirow[t]{2}{*}{$\begin{array}{l}\text { No, de } \\
\text { Iranive? }\end{array}$} & \multirow[t]{2}{*}{ Age } & \multirow[t]{2}{*}{ PoIds } & \multirow[t]{2}{*}{ Synņtômes } & \multicolumn{7}{|c|}{$\begin{array}{l}\text { Activité de la cholinestérase en p. } 100 \\
\text { Temps en semaines anrìs lo demier dourhage }\end{array}$} \\
\hline & & & & 1 & 2 & 3 & 4 & 5 & 6 & 7 \\
\hline $\begin{array}{l}\text { Groupe I } \\
\text { B } 12\end{array}$ & 24 mois & $300 \mathrm{~kg}$ & - & & $2 ?$ & 29 & & & 59 & \\
\hline B 13 & 19 & 250 & - & & 11 & & & & 46 & 33 \\
\hline v 4 & 10 & 210 & - & & & 53 & & & 89 & \\
\hline $\begin{array}{l}\text { Grouve II } \\
\text { B } 19\end{array}$ & 12. & 90 & + & & 10 & & 47 & & & 49 \\
\hline В 24 & 11 & 110 & + & & 41 & & & & 62 & 70 \\
\hline B 40 & 13 & 120 & + & & 25 & & 39 & & 54 & \\
\hline B 41 & 12 & 120 & + & & & 12 & & & 66 & \\
\hline V 5 & 9 & 120 & + & & 22 & 59 & & & & 51 \\
\hline V 7 & 7 & 100 & ++ & & 0 & 6 & 21 & & & 45 \\
\hline V 8 & 6 & 90 & + & 3 & 3 & & 18 & & & $27: 35^{(3)}$ \\
\hline v 11 & 14 & 120 & +++ & & 3 & & 49 & & & 54 \\
\hline V 17 & 12 & 50 & + & & & $16 ; 29^{(3)}$ & & & & 65 \\
\hline A $52(1)$ & 36 & 400 & \pm & & 27 & & & 82 & & \\
\hline v $26(1)$ & Nonvervi-ré & 35 & \pm & 3 & & 73 & & & & 67 \\
\hline $\begin{array}{l}\text { Anima non } \\
\text { traités } \\
\text { I }\end{array}$ & 18 & 250 & - & & 100 & & & & & \\
\hline II & 9 & 150 & - & & 85 & & & & & \\
\hline B 20 & 12 & 200 & - & 23 & & & & 100 & & \\
\hline$v 5(2)$ & 17 & 170 & - & 45 & & 59 & & & & 69 \\
\hline
\end{tabular}

(1) AnImin douché seulement 3 foir.

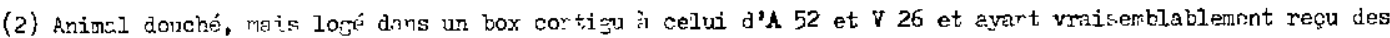
- claboussures d'insecticzde.

(3) 2 Andyses ont f́ti faltes dars une seraine, avec un interville de ó cours nour $\mathrm{V} 8$ et de 5 jours pour $\mathrm{V} 17$. 
tant pour les anımaux, que pour les manipulateurs. L'on utilise trop de produits dont la toxicité à long terme sur les animaux est insuffisamment éprouvée; des expériences de toxicité aiguë ne suffisent pas. Rappelons que KALOW et MARTON (1961) ont montré que le malathion, organophosphoré pourtant pratıquement dépourvu de toxicité aıgue, provoquait des effets nuisibles sur la génération de rats issue d'anımaux qui avalent ingéré le produit, sans montrer eux-mêmes de symptôme d'intoxication. BARNES et DENZ (1951) avaient d'ailleurs déjà sıgnalé de tels effets pour le parathion.

Plusieurs insecticides, de toxicité assez impor tante, sont utilısés couramment dans certains pays pour le détiquage des mammifères, sans que l'on semble se soucier des effets toxiques que ces produits pourraient avoır à long terme.
Notons que même le carbophénothion a été étudié dans le détiquage des bovins ! (WEBBER, 1962 (cité par YOUNGER e.a., 1963), DRUMMOND e.a., 1964). II nous semble certain que les bovıns régulièrement détiqués avec certaıns organophosphorés assez toxıques, recommandés et utılisés comme ixodicıdes, ont obligatorrement, durant toute leur vie, un taux de cholinestérase bien au-dessous de la normale. Les effets à long terme d'une hypocholinestérasémie chronique sont peu connus; nous pensons ici également aux expériences de KALOW et MARTON, au sujet de l'effet sur la génération suivante; ces expériences méritent d'être reprises sur les anımaux domestiques.

Nous remercions Mlle D. CAYRIER de sa collaboration technique.

\section{SUMMARY}

Accidental bovine poisoning by spraying with an organophosphorous insecticide, carbophenothion

21 caitle, 6 months to 2 years old, were sprayed by mistake with carbophenothion at a concentration of 0,1 p 100.18 cattle became ill, after having been sprayed from 4 to 8 times; 9 of these died. The most striking symptoms of this slowly developing inloxication were a profuse diarrhoea, an excessive salivation and a stiff gait. The only constant post-mortem lesion was cedema of the lungs. Age by itself seemed to be less important for individual sensitivity than size and physical condition A kitten died one day after application of a powder contaning 1 p 100 carbophenolhion in talc powder. Results of tests on blood-cholinesterase are given, as well as some remarks on treatment. There was no clear correlation between the importance of symptoms and that of the activity of cholinesterase. The authors warn against the use on animals of insecticides of which long-term toxicity is insufficiently known.

\section{RESUMEN}

\section{Intoxicación accidental de bovinos mediante ducha con un insecticida organofosforado, el carbofenothion}

Por consiguiente de una equivocación, 21 bovinos de edad de 6 meses a 2 años fueron duchados 2 veces por semana, durante casi un mes, con el carbofenothion de una concentración de 0,1 p. 100. 18 bovinos enfermaron, después de 4 a 8 duchas, 9 de ellos murieron.

Una diarrea profusa, una salivación excesiva y un andar rigido constituyen las sintomas más caracterisłıcas de esta intoxicación con evolución lenta. La unica lesión constante a la autopsia era un edema pulmonar. La edad en si parece menos importante para la sensibilidad individual que la talla y la condición fisıca. Se espolvoreó con carbofenothion a 1 por 100 en talco un gatito que murió el dia siguiente Se nolan, Los resultados de las determınaciones de la colinesterasis sanguinea, ası como algunas observaciones en cuanto al tratamiento. No existıa correlación neta entre la importancia de las sintomas y la de la disminución de la actividad de la colinesterasis.

Los autores advierten del peligro, en los anımales, del uso de insecticidos cuya ulteriora toxicidad está conocida insuficientemente. 


\section{BIBLIOGRAPHIE}

1. BARNES (J.M.), DENZ (F. A.). - The chronic toxicity of $p$-nitrophenyl diethyl thiophosphate (E. 605). A long-term feeding experiment with rats. J. Hyg. Camb., 1951, $49: 430-$ 441.

2. BRADY (U. E.), DOROUGH (H. W.), ARTHUR (B. W.). - Selective toxicity and animal systemic effectiveness of several organophosphates. J. econ. Ent., 1960, $53: 6-8$.

3. BRODEUR (J.), DUBOIS (K. P.). - Comparison of acute toxicity of anticholinesterase jnsecticides to weanling and adult male rats. Proc. Soc. exp. Biol. N. Y. 1963, 114 : 509-511.

4. CHARY (R.), JAYOT (R.), BOCQUET (P.). Contribution à la toxicologie du bétail. Titrage de l'activité cholinestérasique sanguine des bovins. Bull. Acad. vét. Fr., 1961, $34: 167-174$.

5. DRUMMOND (R. O.), GRAHAM (O. H.), MELENEY (W. P.), DIAMANT (G.). - Field tests in Mexico with new insecticides and arsenic for the control of Boophilus ticks on cattle. J. econ. ent., 1964, $57:$ 340-346.

6. GAINES (Th. B.). - The acute toxicity of pesticides to rats. Toxical. appl. Pharm. 1960, 2 : 88-99.

7. KALOW (W.), MARTON (A.). - Secondgeneration toxicity of Malathion in rats. Nature, Lond., 1961, 192 : 464-465.

8. Rapport annuel du Laboratoire Central de l'Elevage. Tananarive, 1962.

9. TILEMANS (E. M.). - Quelques insecticides nouveaux et applications récentes. Bull. agric. Congo beige, 1957, 48 : 657-696.

10. WYNIGER (R.). - Appendix to pests of crops in warm climates and their control. $V$. Control measures. Verlag fur Recht und GeselIschoft AG. Basel, 1962: 12.

11. YOUNGER (R. L.), RADELEFF (R. D.), JACKSON (J. B.). - Preliminary studies of the foxicity of carbophenothion and methyl trithion in livestock. J. econ. Ent. 1963, 56 : 757-759. 